\title{
NETest Liquid Biopsy Is Diagnostic of Lung Neuroendocrine Tumors and Identifies Progressive Disease
}

\author{
Anna Malczewska ${ }^{a, b}$ Kjell Oberg $^{c}$ Lisa Bodei $^{d}$ Harry Aslanian $^{a}$ \\ Anna Lewczuk $^{\mathrm{e}}$ Pier Luigi Filosso ${ }^{f}$ Monika Wójcik-Giertuga ${ }^{b}$ \\ Mateusz Rydel $^{\mathrm{b}} \quad$ Izabela Zielińska-Leśb ${ }^{\mathrm{b}} \quad$ Agata Walter $^{\mathrm{b}} \quad$ Alejandro L. Suarez $^{\mathrm{a}}$ \\ Agnieszka Kolasińska-Ćwikłag ${ }^{9}$ Matteo Roffinella ${ }^{f}$ Priya Jamidar ${ }^{a}$ \\ Dariusz Ziora $^{b}$ Damian Czyżewski ${ }^{b}$ Beata Kos-Kudłab Jaroslaw Ćwikłah \\ a Yale University School of Medicine, New Haven, CT, USA; ${ }^{b}$ Medical University of Silesia, Katowice, Poland; \\ 'University Hospital, Uppsala, Sweden; ${ }^{\mathrm{d}}$ Memorial Sloan Kettering Cancer Center, New York, NY, USA; ${ }^{\mathrm{e}}$ Medical

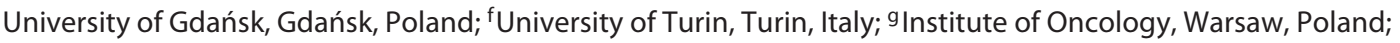 \\ h University of Warmia and Mazury, Olsztyn, Poland
}

\section{Keywords}

NETest - Bronchopulmonary carcinoid - Neuroendocrine tumor - Lung cancer - Transcript · Progression - Biomarker . PCR $\cdot$ Blood

\begin{abstract}
Background: There are no effective biomarkers for the management of bronchopulmonary carcinoids (BPC). We examined the utility of a neuroendocrine multigene transcript "liquid biopsy" (NETest) in BPC for diagnosis and monitoring of the disease status. Aim: To independently validate the utility of the NETest in diagnosis and management of BPC in a multicenter, multinational, blinded study. Material and Methods: The study cohorts assessed were BPC $(n=99)$, healthy controls ( $n=102)$, other lung neoplasia $(n=101)$ including adenocarcinomas (ACC) $(n=41)$, squamous cell carcinomas (SCC) ( $n=37)$, small-cell lung cancer (SCLC) $(n=16)$, large-cell neuroendocrine carcinoma (LCNEC) $(n=7)$, and idiopathic pulmonary fibrosis (IPF) $(n=50)$. BPC were histologically classified as typical (TC) $(n=62)$ and atypical carci-
\end{abstract}

\section{KARGER}

(c) 2019 S. Karger AG, Basel

E-Mail karger@karger.com

www.karger.com/nen noids (AC) ( $n=37)$. BPC disease status determination was based on imaging and RECIST 1.1. NETest diagnostic metrics and disease status accuracy were evaluated. The upper limit of normal (NETest) was 20. Twenty matched tissue-blood pairs were also evaluated. Data are means \pm SD. Results: NETest levels were significantly increased in BPC (45 \pm 25$)$ versus controls $(9 \pm 8 ; p<0.0001)$. The area under the ROC curve was $0.96 \pm 0.01$. Accuracy, sensitivity, and specificity were: 92,84 , and $100 \%$. NETest was also elevated in SCLC $(42 \pm 32)$ and LCNEC $(28 \pm 7)$. NETest accurately distinguished progressive $(61 \pm 26)$ from stable disease $(35.5 \pm 18$; $p<$ 0.0001). In BPC, NETest levels were elevated in metastatic disease irrespective of histology (AC: $p<0.02 ; \mathrm{TC}: p=0.0006$ ). In nonendocrine lung cancers, ACC (18 \pm 21$)$ and SCC (12 \pm $11)$ and benign disease (IPF) $(18 \pm 25)$ levels were significantly lower compared to BPC level $(p<0.001)$. Significant correlations were evident between paired tumor and blood

Prof. Kjell Öberg, MD, PhD

University Hospital

SE-75185 Uppsala (Sweden)

E-Mail kjell.oberg@medsci.uu.se

Lisa Bodei, MD, PhD

Molecular Imaging and Therapy Service, Department of Radiology Memorial Sloan Kettering Cancer Center, 1275 York Avenue, Box 77 New York, NY 10065 (USA)

E-Mail bodeil@mskcc.org 
samples for BPC $(R: 0.83, p<0.0001)$ and SCLC $(R: 0.68)$ but not for SCC and ACC (R: 0.25-0.31). Conclusions: Elevated NETest levels are indicative of lung neuroendocrine neoplasia. NETest levels correlate with tumor tissue and imaging and accurately define clinical progression.

(c) 2019 S. Karger AG, Basel

\section{Introduction}

Bronchopulmonary neuroendocrine neoplasia comprises a spectrum of tumors that arise from pulmonary neuroendocrine cells and represent $\sim 25 \%$ of primary lung neoplasia. Pulmonary carcinoids comprise $~ 2 \%$ of primary lung tumors and $\sim 30 \%$ of neuroendocrine tumors (NET) [1, 2]. The majority of lung carcinoids are identified serendipitously on chest radiology (coin lesions) [2], and even anatomical imaging rarely identifies a mass lesion as a NET [2]. Functional imaging $\left({ }^{68} \mathrm{Ga}-\right.$ labeled somatostatin analog, SSA, PET/CT) is effective, but its resolution is limited by size and somatostatin receptor expression while accuracy is affected by inflammatory cell uptake [2]. A definitive diagnosis can only be established histologically by biopsy or surgery [3]. This reflects that there is no effective diagnostic blood biomarker. Similarly, disease status cannot be adequately assessed since the sensitivity of imaging has limitations in the identification of early progression [4]. Currently, no blood biomarker exists to monitor disease status other than chromogranin $\mathrm{A}$, which is regarded as inadequate for accuracy and methodological reasons $[2,5]$. A key unmet clinical need is a circulating biomarker for diagnosis and to monitor disease status [6].

Bronchopulmonary neuroendocrine neoplasias are histologically separated into well-differentiated carcinoids and poorly differentiated cancers (small-cell lung cancer, SCLC, and large-cell neuroendocrine carcinoma, LCNEC) [2]. The carcinoid group (BPC) is divided into 2 histological subtypes: typical carcinoids (TC) and atypical carcinoids (AC). Histological differentiation between both types is sometimes difficult in a surgical specimen, and TC and AC cannot accurately be distinguished in a biopsy and cytology [2]. Proliferative index measured by Ki67 labeling is not currently utilized for lung NET grading; however, it affords some prognostic value [7]. The updated 2015 WHO classification for lung NETs proposed Ki67 proliferation rates for TC and AC. Therefore, the use of Ki67 appears to differentiate broadly TC (Ki67 $<5 \%)$ from AC $(<20 \%)$ [7]. However, the significant overlap in Ki67 distribution between TC and AC renders it difficult to differentiate between these subtypes. Repeated assessment is clinically challenging since sampling is invasive and cannot be performed frequently $[3,8]$. In a significant number of instances, TC determined by histology subsequently behaves as AC clinically $[2,9]$. Since the clinical course of these 2 tumor types is significantly different, identification and monitoring by imaging, e.g., CT, has limitations both in terms of accuracy and the potential for cumulative exposure to radiation $[2,3]$. Ideally, a circulating biomarker for "liquid biopsy," which could be assessed repeatedly, would provide an optimal strategy for follow-up management [6].

Recent developments in technology have facilitated the delineation of tumor molecular biology and resulted in the development of sensitive and sophisticated strategies, e.g., circulating tumor DNA measurements, to evaluate tumor molecular information in blood [10-12]. These "liquid biopsies" are effective as diagnostic and prognostic markers in diverse neoplasias of the breast, colon, and prostate, for example [13]. They also provide a strategy that decreases invasive biopsies, defines potential therapeutic targets, and provides real-time monitoring to evaluate disease stability/progress [4]. In addition, they capture numerous aspects of tumor biology on a real-time basis and provide the basis for better delineation of individual tumor biology.

BPC have few mutations [14], and examining the transcriptome of the tumor provides an alternative biomarker identification strategy [15]. The evaluation of gastrointestinal NET transcriptomes enabled the development of a liquid biopsy for small-bowel and pancreatic tumors [16]. A comparison of these transcriptomes with BPC confirmed the similarity of matched tumors and lung neuroendocrine neoplasia cell cultures [16]. A correlation of these tumor transcripts in matched circulating blood accurately $\left(R^{2}>0.8\right)$ represented those identified in BPCs [16].

In order to determine whether a circulating molecular signature is effective in clinical practice, a sample-blinded validation in an independent and well-defined cohort of lung NETs, nonneoplastic and poorly differentiated neoplastic lung disease, and controls requires assessment. In the current investigation, we undertook an external assessment of the value of the NETest for discriminating between individuals with BPC, a variety of lung diseases, and healthy volunteers. Sensitivity, specificity, and accuracy (area under the receiver-operating characteristic curve, AUROC) of the NETest were determined in BPC with reference to healthy individuals, or individuals with neoplastic and nonneoplastic lung disease at centers in 
Italy, Poland, and the USA. We evaluated whether the NETest results could differentiate BPC from controls and distinguish neoplastic and benign disease as well as identify disease status.

\section{Materials and Methods}

\section{Strategy}

We compared circulating NETest levels from BPC to healthy controls as well as patients with idiopathic pulmonary fibrosis (IPF), SCLC, and LCNEC, squamous cell carcinoma (SCC), and adenocarcinomas (ACC). This is a separate patient cohort than the one previously reported $[16,17]$. We calculated diagnostic accuracy and metrics (AUROC, sensitivity, and specificity) for the NETest in each group. We also evaluated the concordance in NETest gene expression in paired blood and tumor tissue samples from BPC, SCLC, SCC, and ACC.

\section{Subjects}

All patients provided informed consent for the blood translational analysis authorized by local ethics committees. This multicenter prospective cohort was studied between June 2017 and March 2018. It included patients and nonaffected family members attending oncology, endocrinology, and pulmonology outpatient clinics. BPC patients with histological confirmation of the disease met the criteria for inclusion. No exclusion criteria were applied. Patient cohorts included healthy controls $(n=102)$ and patients with BPC $(n=99)$ of various grade, histology, disease status, and therapies, including those with no evidence of disease (NED) on imaging, as well as other neuroendocrine and nonneuroendocrine lung diseases (Table 1). This included IPF $(n=50)$; 23 lung neuroendocrine carcinomas (LCNEC: $n=7$; SCLC: $n=16)$; SCC $(n=$ $37)$, and ACC $(n=41)$. Patients with no evidence of residual disease by imaging were included. Control subjects were included if there was no known malignancy present at the time of blood sampling, and they identified themselves as asymptomatic in good health.

BPC $(n=99)$ were histologically classified into TC $(n=62$, $63 \%)$ and $\mathrm{AC}(n=37,37 \%)$ by the institute per standard ENETS (European Neuroendocrine Tumor Society) criteria from which the patients were recruited. Central review was not undertaken. Radiological status - stable (SD) or progressive disease (PD) was assessed by RECIST 1.1 criteria. SD was noted in 78 (including 20 NED). Twenty NED (surgical "cures" [disease free]) confirmed by imaging (20 had CT, 6 had ${ }^{68} \mathrm{Ga}-\mathrm{SSA}$ PET/CT) were histologically classified as TC $(n=15)$ and AC $(n=5)$. The time between surgery and postoperative imaging was $1.8 \pm 2.6$ years (0.75: 0.27-2.12). The majority ( 90\%) were T1 (TC: 75\%; AC: $55 \%$ ) and T2 (TC: $11 \%$; AC: $32 \%$ ). TC (with disease: $n=53$ ) were N0M0 $(n=21), \mathrm{N}+\mathrm{M} 0(n=15)$, and $\mathrm{N} \pm \mathrm{M} 1(n=17)$. For AC (with disease: $n=32)$, the distribution was: N0M0 $(n=6), \mathrm{N}+\mathrm{M} 0$ $(n=11)$, and $\mathrm{N} \pm \mathrm{M} 1(n=15)$. M1 included hepatic and other distant metastases $(n=29,29 \%)$. Of the 21 with PD, $12(52 \%)$ exhibited M1 disease. At the time of blood sampling, 23\% BPC were being treated with capecitabine and temozolomide $(n=2$, $2 \%)$, peptide receptor radionuclide therapy $(n=2,2 \%)$, or SSA $(n=19,19 \%)$.

NETest Validation for BPC

\section{Blood Sampling}

Blood samples (6 mL; peripheral blood) were collected in EDTA tubes, mixed, and immediately stored on ice. Tubes were coded and stored at $-80^{\circ} \mathrm{C}$ within $2 \mathrm{~h}$ of collection per standard molecular diagnostics protocols for PCR-based studies [18]. Randomly selected coded blood samples were sent in mixed batches, blinded, and deidentified to Wren Laboratories, Connecticut, USA. All samples were anonymized and coded, and all investigators were blinded to the blood source, clinical diagnosis, and disease status.

\section{Tumor Tissue Sample Collection}

Tumor tissue ( $n=20$; Table 1 ) was collected at the time of surgery as previously described [16]. Samples were snap frozen in liquid nitrogen. Deidentified samples were sent to Wren Laboratories for RNA isolation and NETest PCR.

\section{NETest}

Details of the PCR methodology, mathematical analysis, and validation have been published in detail, comprising a 2-step protocol (RNA isolation/cDNA production and q-PCR) from EDTAcollected whole blood [16-19]. The assay is undertaken in a US clinically certified laboratory (Wren Laboratories CL-0704, CLIA 07D2081388). Transcripts (mRNA) were isolated from EDTAcollected whole blood samples (mini blood kit; Qiagen, Valencia, CA, USA), and real-time PCR was performed [20] (Fast Universal PCR Master Mix, Life Technologies). Target transcript levels are normalized and quantified versus a population control [17-19]. Final results are expressed as an activity index (NETest score) from 0 to $100 \%$ [17-19]. The upper limit of normal is 20 .

\section{Statistical Analysis}

The required total sample size (NETs and controls, power 0.8 and $\alpha=0.05$ ) to attain significant differences in NETest scores was calculated to be a minimum of 46 patients/subjects in each group.

Intergroup analyses were undertaken using 2-tailed nonparametric tests (Mann-Whitney $U$ test or Wilcoxon signed rank test for paired samples). Receiver-operating characteristic curve (ROC) analysis was used to determine the diagnostic accuracy of the NETest [21-23]. Metrics calculated included sensitivity, specificity, likelihood ratios, the $z$-score (values $>1.96$ are significant), and calculation of the Youden $J$ index (performance of a diagnostic). Prism 6.0 for Windows (GraphPad Software, La Jolla, CA, USA; www.graphpad.com) and MedCalc statistical software version 16.2.1 (MedCalc Software bvba, Ostend, Belgium; http:// www.medcalc.org; 2017) were utilized. Post hoc correction (Dunn's multiple comparison test) for multiple testing was utilized where appropriate.

For tumor:blood pairs, we undertook Pearson regression analysis following log transformation of normalized expression levels. Statistical significance was defined at a value of $p \leq 0.05$. Data are presented as means \pm SD (median: [interquartile ranges]).

\section{Results}

\section{$B P C$ and Controls}

We first evaluated blood gene expression scores in the BPC-control set. The NETest was significantly increased 


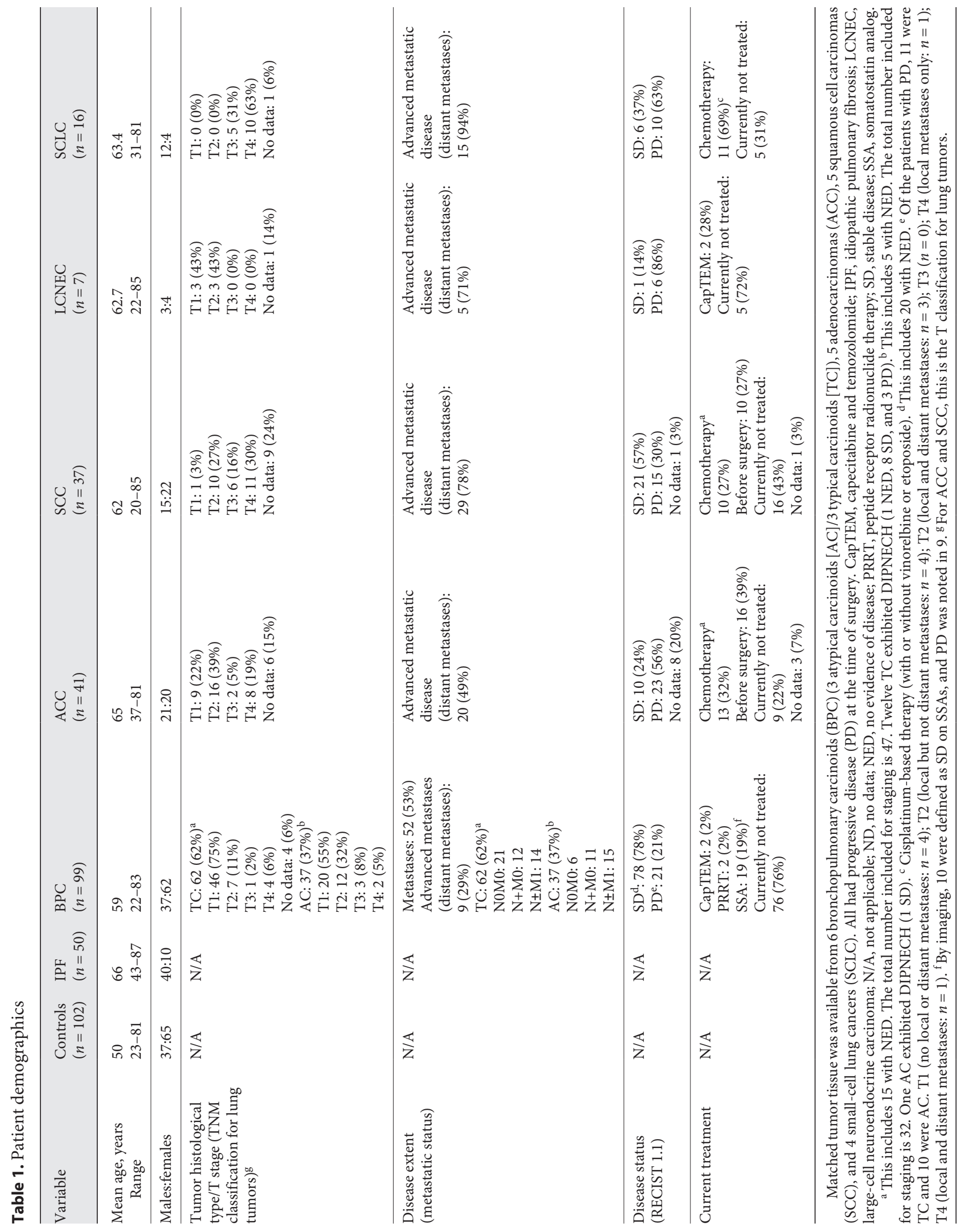


Fig. 1. a NETest in controls and BPC: Measurements of the NETest identified elevated levels in BPC (red) versus controls (blue) $(p<0.0001)$. b AUROC for BPC and controls: the AUROC for differentiating BPC from controls was 0.96 (95\% CI: 0.92-0.98, $p<0.0001)$. Mean \pm SEM.



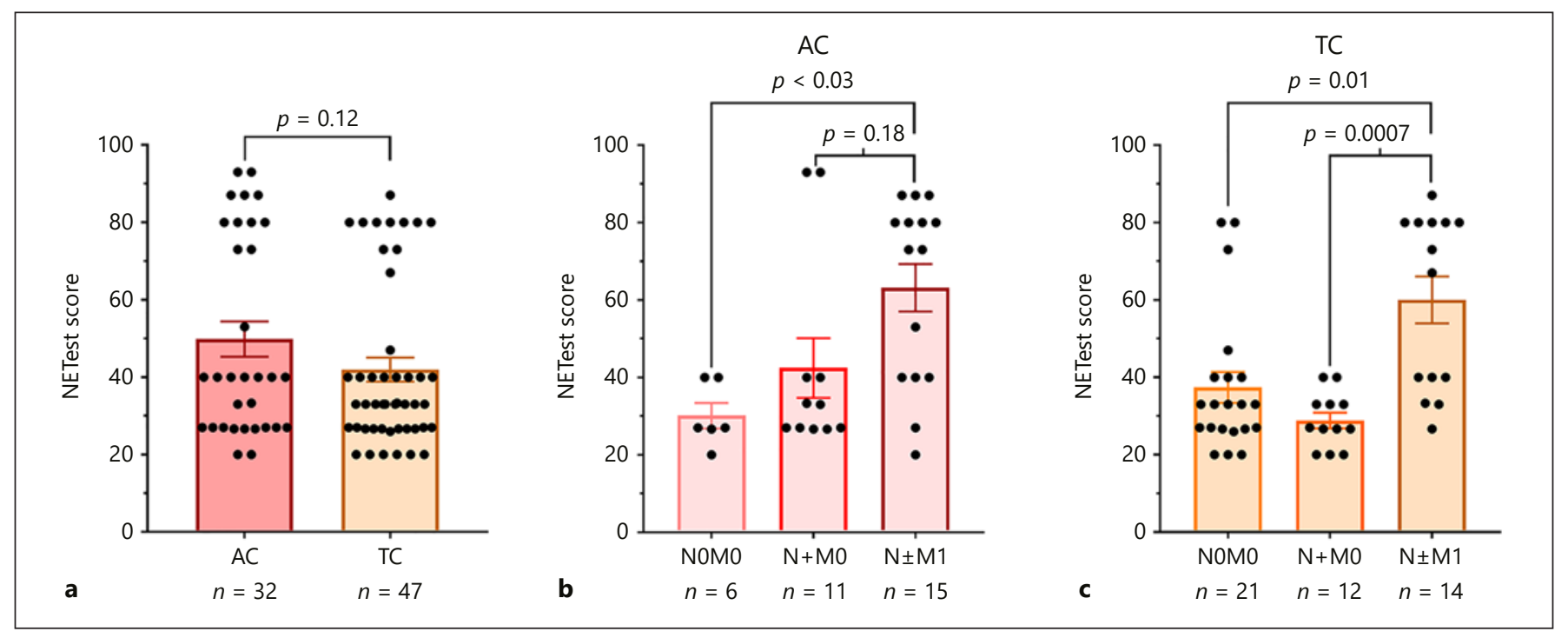

Fig. 2. NETest in $B P C$ - relationship to histological assessment. a NETest in AC and TC with image-detectable disease: measurements of the NETest-identified levels were not significantly elevated in AC (atypical carcinoid) with evidence of disease (red) versus TC (typical carcinoid) with evidence of disease (orange) $(p=0.12)$. b NETest in AC by stage: NETest levels were significantly elevated in AC with distant metastases (M1) compared to AC with no metastatic disease (N0M0) $(p<0.03)$. Levels were not significantly elevated compared to those with lymph node disease $(\mathrm{N}+\mathrm{M} 0)$ but no distant metastases $(p=0.18)$. Group analysis identified NETest was significantly different across all 3 groups $(p<0.02)$. c NETest in TC by stage: NETest was significantly elevated in TC with distant metastases (M1) compared to those without metastatic disease (N0M0) $(p=0.01)$ and with lymph node disease $(\mathrm{N}+\mathrm{M} 0)$ but no distant metastases $(p<0.001)$. Group analysis identified NETest was significantly different across all 3 groups $(p=0.0006)$. Means \pm SEM. in BPC (44.5 \pm 25 [33:27-73]) compared to controls ( $9 \pm$ 8 [7: 0-20], $p<0.0001$ ) (Fig. 1a). AUROC analysis value was $0.96 \pm 0.01$ (Fig. 1b). The $z$-statistic was 41.6 and the Youden index J was $82.3 \%$. The accuracy, sensitivity, and specificity at a NETest cutoff of 20 was: 92,84 , and $100 \%$. No controls exhibited a score $>20 \%$.

Of BPC $(n=20)$ classified as NED by imaging $(20 / 20$ CT-negative, $6 / 6{ }^{68} \mathrm{Ga}$-SSA PET/CT negative), 18 (90\%) exhibited scores $\leq 20 \%$. Two asymptomatic patients with negative imaging exhibited scores of 33 and 40, respectively. Subset analysis identified that patients with NED $(n=20)$ exhibited significantly lower $(p<0.0001)$ NETest scores (24 \pm 6 [27: 20-27]) than patients with proven disease $(n=79 ; 45 \pm 23$ [40:20-73]).

Excluding this group (NED) from the AUROC analysis identified a value of $0.98 \pm 0.01$. The $z$-statistic was 


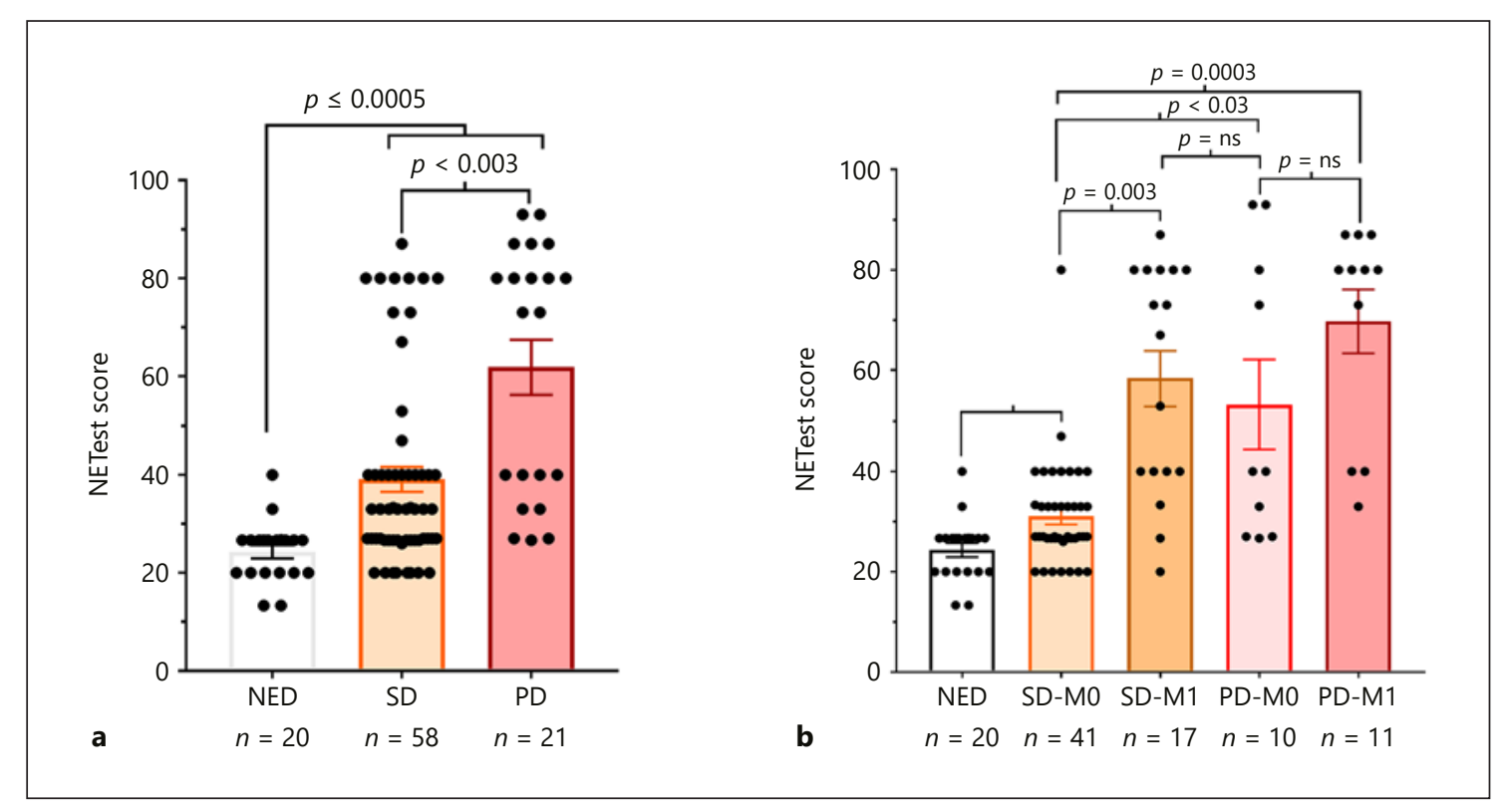

Fig. 3. NETest in BPC - relationship to clinical status and metastatic disease. a Disease status (RECIST based): measurements of the NETest-identified levels were significantly elevated $(p<0.0005)$ in stable disease (SD - orange) and progressive disease (PD - red) compared to no evidence of disease (NED) "surgical cures" (white). Levels were significantly elevated $(p=0.0026)$ in PD compared to SD. b Metastatic disease: NETest levels were significantly elevated

79.3, and the Youden index J was 90\%. The sensitivity for differentiating BPC with evidence of disease from controls was $90 \%$ with a specificity of $100 \%$ using a NETest cutoff of 20 .

Utilizing histological classification, NETest levels for TC with evidence of disease $(n=47)(42 \pm 21$ [33:27-47]) and AC $(50 \pm 26$ [40:27-80]) $(n=32)$ were overall not significantly different $(p=0.12$ ) (Fig. 2a). A subset analysis of T stage identified no relationship between size and the NETest (Kruskal-Wallis test). Analysis of the disease extent (metastatic status) using TNM staging criteria identified that AC with M1 disease exhibited a significantly higher score $(63 \pm 24$ [73: 40-80]) than patients with no metastases ( $30 \pm 8$ [27: 25-40]) $(p<0.03$, Dunn's test) (Fig. 2b). Overall, the scores were significantly different between those with no metastases, those with lymph node metastases, and those with distant metastases \pm lymph node metastases (Kruskal-Wallis test 7.84, $p<0.02)$. TC with M1 disease exhibited a significantly higher score $(60 \pm 23$ [70: 38-80]) than no metastases (37 \pm 18 [33: 27-40]) ( $p=0.0007$, Dunn's test) (Fig. 2c). Overall, the scores were significantly different between stages (no metastases, lymph node metastases, and dis- in SD with no evidence of distant metastases (SD-M0) compared to NED $(p<0.03)$. Levels were elevated in SD with distant metastases (SD-M1) versus SD-M0 $(p=0.003)$. Levels were similarly elevated in PD with (PD-M1) and without metastases (PD-M0). Both PD groups were significantly elevated versus SD-M0 ( $p=$ 0.03; $p<0.0003$, respectively). Means \pm SEM.

tant metastases \pm lymph node metastases) (Kruskal-Wallis test $14.78, p=0.0006$ ). An evaluation of M1 versus locoregional metastases or no metastases, irrespective of histological classification, identified that scores were significantly elevated ( $p<0.0001$, Dunn's test) in M1 (62 \pm 23 [73: 40-80]) compared to M0 (36 \pm 18 [30: 27-40]).

In $13 \mathrm{BPC}$, there was evidence of diffuse idiopathic pulmonary neuroendocrine cell hyperplasia (DIPNECH), AC: $n=1$ and TC: $n=12$. Scores were not significantly different between those with DIPNECH $(39 \pm 22$ [47: 27$40])$ and those without $(37 \pm 18[33: 27-40])(p=0.10)$.

An evaluation of disease status (radiologically documented at the time of blood sampling) identified those with PD $(61 \pm 26$ [73: 33-80]) to exhibit significantly higher levels ( $p=0.0026$, Dunn's test) than those with SD (35.5 \pm 18 [27: 27-40]) (Fig. 3a). Levels for both PD and SD were significantly elevated versus NED $(p<0.0005$, Dunn's test).

Stratifying NETest scores by disease status and presence of distant metastases identified that individuals with NED or imaging-documented SD but without distant metastases exhibited the lowest scores (31 \pm 10 [27: 2733]) (Fig. 3b). Levels in SD-M0 patients were, however, 
Fig. 4. NETest in IPF and BPC. a NETest in controls, IPF, and BPC: NETest levels were not significantly elevated in IPF versus controls $(p=0.23)$. Levels were significantly $(p<0.0001)$ lower than in BPC. b AUROC for IPF and BPC: the AUROC value differentiating BPC from IPF was 0.81 (95\% CI: 0.74-0.87, $p<0.0001$ ). Means \pm SEM.

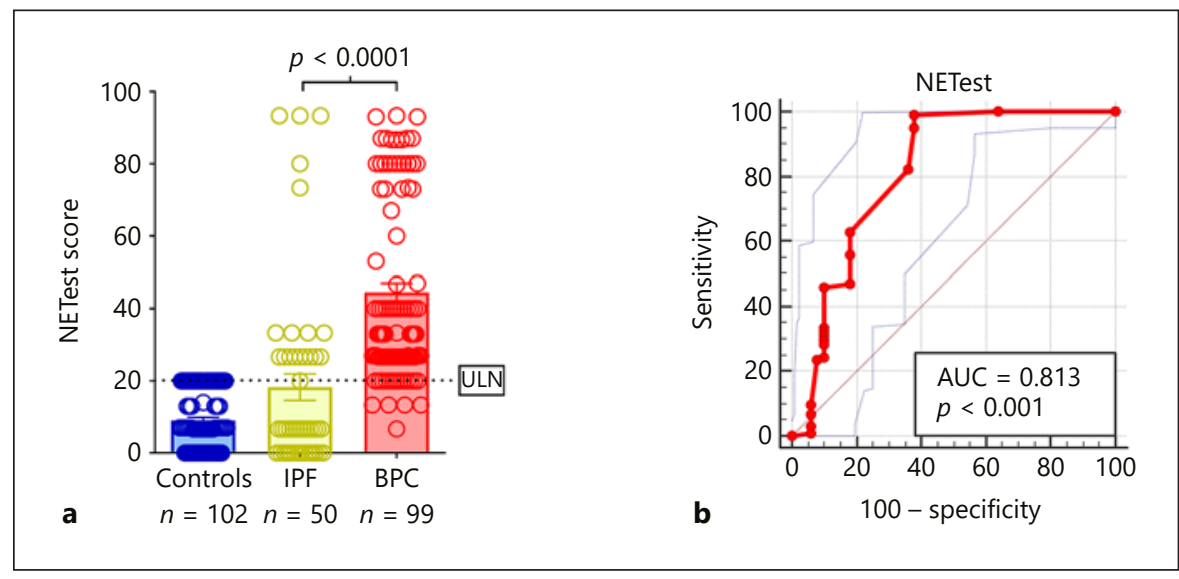

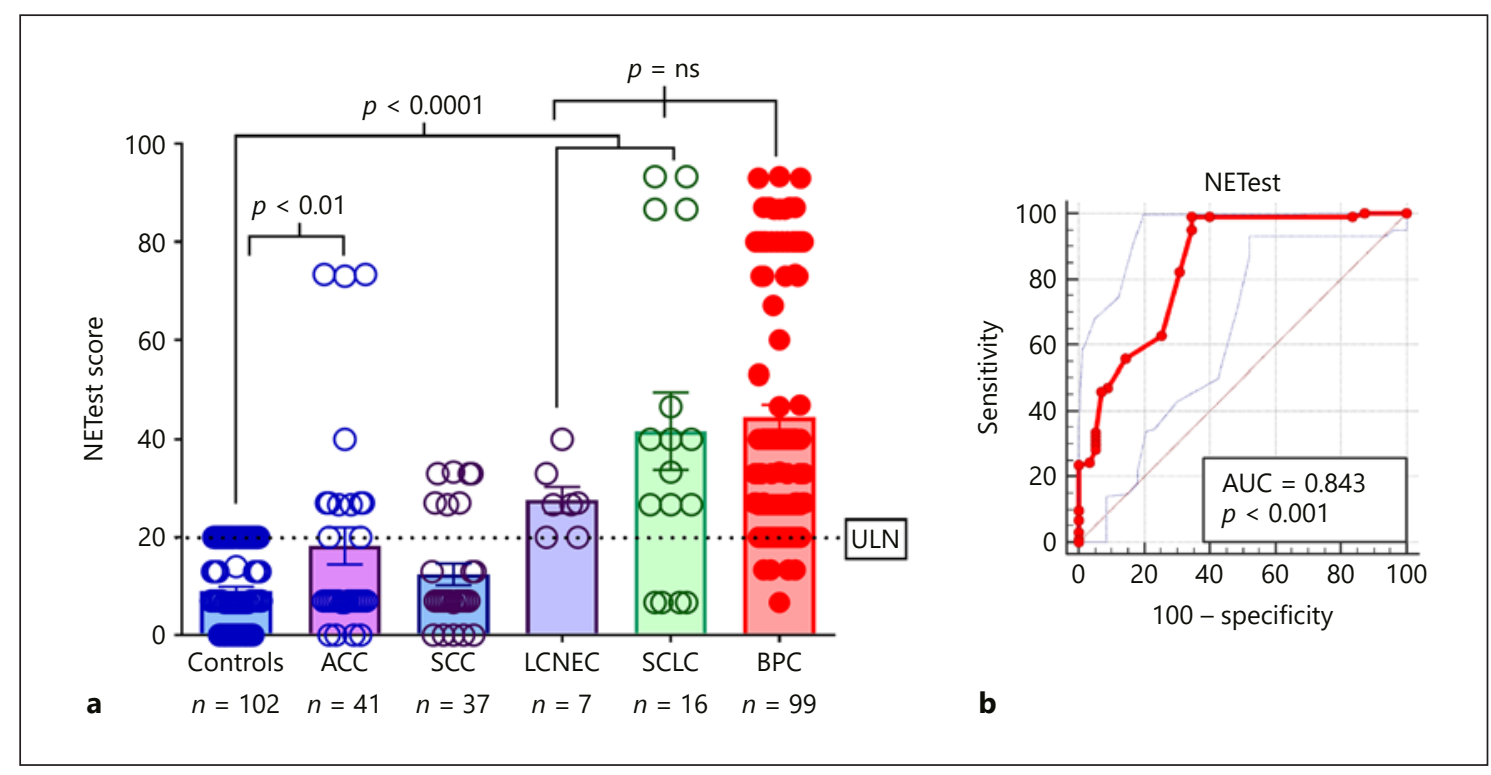

Fig. 5. NETest in other malignant lung diseases (non-BPC). a NETest in controls and different lung neoplasias: NETest levels were elevated in SCLC $(p<0.0001)$, LCNEC $(p<0.0001)$, and ACC $(p=0.0067)$. SCC levels were not elevated versus controls $(p=0.09)$. Both ACC and SCC had significantly lower expression than BPC $(p<0.0001)$. No differences were noted between BPC and either

higher than in patients with NED ( $p<0.03$, Dunn's test). SD patients with M1 disease exhibited significantly higher scores (58 \pm 23 [67:40-80]) than those without metastases ( $p=0.003$, Dunn's test). Levels in individuals with PD were similarly elevated (PD-M0: $53 \pm 28$ [40:27-83]; PD-M1: $70 \pm 21$ [80: 40-87]). Scores did not significantly differ between SD-M1 and PD-M0 or PD-M1. Scores were, however, significantly elevated in PD-M0 $(p<0.05$, Dunn's test) and PD-M1 ( $p=0.0003$, Dunn's test $)$ compared to SD-M0.
SCLC $(p=0.80)$ or LCNEC $(p=0.12)$. b AUROC for BPC and ACC/SCC: the AUROC value for differentiating BPC from ACC/ SCC (as a group) was 0.84 ( $95 \%$ CI: $0.78-0.90, p<0.0001$ ). No difference was noted for SCLC and LCNEC versus BPC (AUROC: $0.56, p=0.31)$. The difference between SCLC/LCNEC versus ACC/ SCC (AUROC: $0.74, p=0.0002$ ) was significant. Means \pm SEM.

Stratifying the scores by histology identified no significant relationship between AC or TC and disease progression. While the proportion of patients who had PD was higher in the AC group (10 of 32,31\%), this did not reach statistical significance (TC: 11 of 47, 23\%) (Fisher's exact test: $p=0.45)$. Patients with SD-M1 or PD-M0 also had similar proportions of AC (40-47\%) and TC (53$60 \%)$.

Stratifying the scores by SSA therapy, the predominant therapy used in this study, identified a significant rela- 




Fig. 6. NETest gene expression in matched tumor tissue:blood samples from malignant lung diseases. Linear regression (Pearson) analysis of log-transformed normalized values of each of the individual tumor-blood pairs identified $R$ to range from 0.82 (BPC; $n=6, p<0.0001$ ) to 0.68 (SCLC; $n=4, p<0.0001$ ) to 0.25 (ACC; $n=5, p=0.12$ ) to 0.31 (SCC; $n=5, p=0.06$ ). In the graph plots, the pairs (blood-tissue) were averaged, and error bars indicate standard errors of the mean. The dashed line represents the best linear fit to each dataset. tionship between therapy use and disease status. PD patients treated with SSA $(n=9)$ exhibited significantly higher scores (76 \pm 20 [80:73-87]) than those who had $\mathrm{SD}(n=10: 38 \pm 21[33: 26-43])(p=0.0035)$.

\section{Gene Expression in Benign Lung Disease}

Gene expression scores were determined in IPF patients to investigate whether lung fibrosis altered the score since this pathobiology has been identified in BPC and is often a related sequela of carcinoid disease.

IPF NETest levels were not significantly increased $(18 \pm 25$ [7: 0-27] $)(p=0.23)$ versus controls (Fig. 4a). Levels were less than noted in BPC $(p<0.0001)$. The AUROC (vs. BPC) was $0.81 \pm 0.04$ ( $p<0.001)$ (Fig. 4b). Elevated IPF scores occurred in 18 individuals, of whom 5 (10\%) exhibited levels $>70 \%$. 
Gene Expression in Other Malignant Lung Diseases

Gene expression scores were also determined in other malignant diseases. We assessed both neuroendocrine lung neoplasia (SCLC and LCNEC) and nonneuroendocrine neoplasia (ACC and SCC) to examine the specificity of the BPC signature.

NET gene expression levels were elevated in SCLC $(42 \pm 32$ [37: 12-77]) $(p<0.001)$ and LCNEC $(28 \pm 7$ [27: $20-47])(p<0.001)$ versus controls. Both of these tumor types exhibited neuroendocrine neoplastic phenotypes (Fig. 5a). NETest levels were elevated (>20\%) in $75 \%$ of SCLC and $62.5 \%$ of LCNEC. No difference in NETest levels was noted for SCLC and LCNEC versus BPC (AUROC: $0.56, p=0.31$ ). A subanalysis comparing AC and either SCLC or LCNEC identified AUROC of 0.52 $(p=0.83)$ and $0.65(p=0.14)$, respectively.

SCC NETest levels (12 \pm 11 [7: 7-27]) were not significantly elevated versus controls $(p=0.09)$. Levels were, however, increased in ACC (18 \pm 21 [7: 7-27]) $(p=$ $0.0067)$ versus controls. The NETest was, however, significantly higher $(p<0.0001)$ in BPC than in SCC and ACC. AUROC (vs. BPC) was $0.84 \pm 0.03(p<0.001)$ (Fig. 5b). Using a NETest cutoff of 13, the sensitivity for differentiating BPC from ACC/SCC was $100 \%$, but the specificity was $67 \%$. Increasing the cutoff to 27 resulted in a sensitivity and specificity of $75 \%$. Twenty-six to $29 \%$ of SCC and ACC exhibited NETest levels $>20 \%$. Overall, the difference between tumors with a neuroendocrine (SCLC/ LCNEC) versus nonneuroendocrine phenotype (ACC/ SCC) was significant (AUROC: $0.74, p=0.0002$ ).

Gene Expression in Matched Blood and Tumor Tissue

Finally, we evaluated the concordance between gene expression in matched tumor tissue and blood sample pairs $(n=20)$. We evaluated BPC $(n=6)$, SCLC $(n=4)$, SCC $(n=5)$, and ACC $(n=5)$. The Pearson correlation $(R)$ for normalized gene expression in each of the tumorblood pair groups ranged from 0.82 (BPC) and 0.68 (SCLC) to 0.31 (SCC) and 0.25 (ACC) (Fig. 6). Significant correlations between tumor and blood were only demonstrable for BPC $(p<0.0001)$ and SCLC $(p<0.0001)$. Correlations were not significant for SCC $(p=0.06)$ and ACC $(p=0.12)$.

\section{Discussion}

The expression of a NET multigene signature has been demonstrated to be consistently detectable in the blood of BPC patients $[16,17]$. The current study was designed to assess this observation and independently validate it in an independent cohort of European and North American individuals comprised of subjects from Poland, Italy, and the USA. All samples were collected using an identical protocol under "real-world" conditions. Laboratory analysis of deidentified samples was undertaken by personnel blinded to the source of blood and unaware of any clinical details. Clinical correlations of control, lung disease, and $\mathrm{BPC}$ groups were undertaken by blinded investigators to prevent observer bias. Limitations of the study include the lack of central review for histology, heterogeneity of some of the groups studied, the relatively small numbers available for evaluation in some of the subgroups, incomplete functional imaging data on all patients, and the absence of follow-up to address the prognostic significance of the NETest. In addition, once adequate sample numbers are available, it seems likely that to optimize the NETest for other lung neuroendocrine neoplasia (e.g., SCLC) some remodeling of the algorithmic reconfiguration will be necessary.

This real-world study of the utility of the NETest in lung neuroendocrine neoplasia confirmed that the NETest was accurate and specific for bronchopulmonary neuroendocrine neoplasia. In addition, the NETest could differentiate BPC with residual disease from those who were surgically "cured." Furthermore, the NETest could discriminate both AC and TC with metastatic disease from those with localized disease. The NETest was significantly higher in BPC with PD, especially if metastatic. Finally, gene expression was highly correlated (tissue to blood) in lung tumors with a neuroendocrine phenotype. In lung cancers without a neuroendocrine profile, there was no correlation. The use of this "liquid biopsy" may provide an appropriate method to diagnose BPC and facilitate the assessment of disease status.

In our study, the mean NETest level in 99 BPC was $45 \%$, while it was $9 \%$ in controls. This is consistent with previously reported data where the mean NETest was $48 \%$ in BPC and $6 \%$ in controls [17]. The AUROC for differentiating BPC from controls of 0.96 is comparable to the 0.98 previously reported [17]. Thus, the multigene assay is accurate at distinguishing neuroendocrine disease from controls. In the current study, sensitivity of the NETest was $84 \%$, which is lower than the previously identified sensitivity of $93 \%$. This may reflect the high proportion $(20 \%)$ of surgically cured patients in the current cohort.

The evaluation of the surgically treated patients is of interest since the low NETest values in this group may have contributed to the overall decrease in diagnostic sen- 
sitivity of the NETest. In this group (15 TC, 5 AC), 18 had normal NETest values, of whom 15 were TC and 3 were AC. Excluding this group (NED) from the analysis provided a sensitivity for differentiating BPC from controls of $98 \%$. The high proportion (90\%) of these patients who had undergone an R0 resection (and had normal values postoperatively; mean 1.8 years after surgery) suggests that the NETest may have value as a biomarker of "complete" surgical resection. The $2 \mathrm{AC}$ patients with elevated levels who currently have no imaging-based evidence of disease suggest that the NETest may have identified nonimage-detectable disease. It has previously been reported that initial image-negative NETest scores have subsequently been identified to have been "true positives" when image-positive conversion was noted during the 2 years after surgery $[17,24]$. It has been suggested that measurement of transcript levels before and after BPC surgery will facilitate early identification of residual or recurrent disease prior to imaging. This has been demonstrated to be the case in gastroenteropancreatic NET disease [18].

NETest levels were not related to BPC histology. We were unable to identify significant differences $(p=0.12)$ in those with AC (mean 50) compared with TC (mean 42). This suggests that the NETest levels do not correlate with the reported tumor tissue pathology. It is well recognized that a percentage of histologically classified TC subsequently present with recurrent or metastatic disease [2, 5]. Furthermore, the classification of endocrine tumor disease as benign or malignant is recognized as difficult even amongst experts although $\sim 70 \%$ of cases are properly identified [25]. However, both AC and TC with metastatic disease exhibited significantly higher scores than those with localized disease. This suggests that the elevated NETest scores may reflect either evidence of altered proliferation rates or increased tumor burden. A possible explanation might be the high percentage (AC: $41 \%$ and TC: $23 \%$ ) of M1 patients in each of the 2 histological categories (Fisher's 2-tailed test: $p=0.07$ ). The overall lack of difference between AC and TC probably reflects more complex issues which may be better evaluated in the future by an in depth "omic" analysis of individual tumors.

In 13 individuals with BPC, the coexistence of DIPNECH was identified. NETest levels were similar in this cohort (mean 39) compared to those with no DIPNECH (mean 37). Separate studies of DIPNECH are needed to define the role of the NETest in the delineation of this ill-understood pathobiological condition. This lack of difference may reflect the current understanding of DIPNECH as a subset of peripheral carcinoid tumors with low malignant potential [26].

NETest levels have been reported to be of value in defining SD and PD $[18,19]$. In this study, we identified significantly $(p<0.0001)$ higher levels in PD (mean: 61) than SD (mean: 35$)$. The AUROC value for differentiating both clinical groups was $0.79(p<0.0001)$. The mean NETest levels are similar to previous publications in BPC with PD: 73-85 [16, 17]; SD: 32-36 [16, 17]; and AUROC: 0.91 [17]. We also noted that effective therapy, e.g., SSA, was associated with lower scores. Patients on SSA who were "responding" i.e., exhibited SD by imaging, exhibited a significantly $(p<0.005)$ lower score (mean: 38$)$ than those with image-demonstrable progression (mean score: 76). This is consistent with a recent study demonstrating that the NETest effectively identified SSA-treated patients who are responding to therapy [27].

In the current study, we also examined whether disease stage (evidence of distant metastases) was related to higher scores in the PD cohort. Levels in PD with distant metastases $(70 \pm 21)$ were not significantly different to those without (53 \pm 28$)$. This observation is partially supported by our observations that "stable" patients with M1 disease had a significantly higher level $(58 \pm 23)$ than patients who were stable and had no metastatic disease (31 \pm 10$)$. It is possible that specific omic analysis of individual tumors and their metastases may allow a better delineation of what constitutes PD in a metastatic setting. Such alterations in the molecular status and their relationship to disease status have been identified by Alvarez et al. [28]. The NETest may, therefore, provide information useful in defining the malignant potential of a particular tumor, especially once the specific omic clusters delineating the hallmarks of malignancy are identified. Thus, the current study supports previous reports that the NETest can function as a marker or index of clinical disease activity.

In order to assess specificity of the test, we examined whether the NETest identified other lung neuroendocrine neoplasia. Levels of the gene signature were positive in $~ 75 \%$ of SCLC and LCNEC. The mean levels ranged between 28 (LCNEC) and 42 (SCLC). Although there was no significant difference in the AUROC between BPC and LCNEC/SCLC, "omic" analyses [10] identified significantly elevated expression of genes involved in secretion and somatostatin receptors in BPC compared to LCNEC/SCLC. For example, the secretion-associated gene PNMA2 was $>2$-fold higher in BPC than SCLC/ LCNEC; this is consistent with an earlier observation identifying elevated serum PNMA2 expression in TC/AC [29]. SSTR5 was also highly expressed in BPC; this is con- 
sistent with the elevated circulating expression noted in the peripheral blood [30] and the known decreased expression in both SCLC and LCNEC [31]. These data suggest that there are individual differences in gene expression between BPC and SCLC/LCNEC [unpubl. Data: Drozdov et al., 2018]. The NETest algorithms were originally trained to differentiate NETs from controls [20]. Retraining the algorithms on gene expression patterns from BPC and SCLC/LCNEC will likely result in a lung-specific test that can differentiate between these different histological types [32].

The evaluation of geneexpression in tumor tissue:blood pairs supports the NETest signature is neuroendocrine in origin. The significant correlation between tumor and blood levels of the 6 BPC and 4 SCLC (neuroendocrine phenotype)-matched tissue-blood sample pairs confirmed parallel expression of all genes in tissue and blood compartments. The absence of a significant correlation for ACC and SCC demonstrates the NETest is specific for genes expressed by lung neuroendocrine neoplasia. As such, a blood-based gene expression test can function as surrogate marker of tumor tissue expression, i.e., as a "liquid biopsy." A previous report of BPC demonstrated that NETest gene expression levels were highly correlatable between tumor tissue and matched blood ( $R$ : 0.63-0.91, $p<0.001)$ [16]. This current study confirms the significant correlation between blood marker expression and transcript levels in BPC $(R: 0.82, p<0.0001)$ and extends the observation to SCLC which also exhibited a significant correlation $(R: 0.68, p<0.0001)$. We interpret this to reflect the known neuroendocrine tissue phenotype of these tumors which is evident in blood. In contrast, in SCC and ACC, gene expression in tissue and blood was not significantly correlated. This is consistent with information in merged lung cancer transcriptome data sets ( $n=1,118$ individual ACC/SCC tumor transcriptomes) that are currently used for clinical predictive modeling. These transcriptome data sets failed to identify significant expression of NETest genes [33]. Furthermore, an audit of the 42 different tissue-based lung gene expression assays used as prognostic signatures for ACC/SCC [34] failed to identify any NETest genes. These data would appear to confirm that NETest genes are neuroendocrine related. These observations have recently been confirmed by a large NIH-funded study (including gene expression data from 10,224 samples from 32 different tumor types). This categorically demonstrated that the NETest genes captured a neuroendocrine neoplasia phenotype [35]. Overall, gene expression in tumor tissue, irrespective of histology, is recapitulated in time-matched blood sam- ples from BPC and SCLC. Levels of gene expression in blood, therefore, provide accurate and correlatable measurements of lung NET tissue (BPC and SCLC) expression.

Gene expression levels were, however, detectable in blood from nonneuroendocrine neoplastic lung disease, and $\sim 25 \%$ of SCC and ACC exhibited detectable NETest scores. Levels were increased in ACC compared to controls but not in SCC. This is similar to previously reported data [17]. A minority (10\%) of ACC exhibited NETest levels $>40 \%$. This may reflect the well-described phenomenon of neuroendocrine differentiation in highly aggressive lung tumors $[36,37]$ and likely detects a tissuederived neuroendocrine phenotype. Recent publications have identified significant cellular heterogeneity in lung neoplasia including NE differentiation in $~ 10 \%$ of ACC and reclassification of $5 \%$ of SCCs as LCNEC $[36,38,39]$. A mixed neuroendocrine phenotype is associated with a poor prognosis in these tumors [40]. The identification of elevated circulating gene levels in such tumors might consequently be of adjunctive value in more precise disease stratification. In terms of specificity, an AUROC of 0.84 was calculated for BPC versus ACC/SCC suggesting that the test, as currently configured, can discriminate between nonneuroendocrine- and neuroendocrine-derived tumors. Specifically, using an adjusted cutoff of 27 for the NETest was associated with a sensitivity and specificity of $75 \%$ for differentiating BPC from either ACC or SCC.

In addition to malignant lung disease, we evaluated the NETest in IPF patients. A previous report noted that chronic obstructive pulmonary disease had low-level increases in circulating NETest gene expression [17]. We postulate that this increase in gene expression reflects the well-described increase in histological foci of pulmonary neuroendocrine cell proliferation and tumorlets that occur in this disease [41]. A similar process has been described in chronic gastritis with increased ECL cell proliferation and concomitant increases in neuroendocrine gene expression [42]. Since inflammation is a common correlative phenomenon associated with both chronic obstructive pulmonary disease and IPF [43, 44], we evaluated whether idiopathic fibrotic changes in the lung might also elevate the NETest. The NETest was positive in $36 \%$ of IPF patients, but levels were not significantly increased $(p=0.23)$ versus controls. These data are of interest since they suggest a role for inflammation as an activator of NETest gene expression. It should be noted that 5 IPF patients exhibited a NETest $>70 \%$. The high level in a "nonneuroendocrine" disease is consistent with neuroendocrine cell activation but may also reflect occult neuroendocrine disease. This 
possibility is supported by a number of case studies linking BPC to lung fibrosis $[45,46]$.

The measurement of gene expression levels in blood accurately discriminated BPC from controls in this large independent validation study and could also distinguish other bronchopulmonary neoplasias as well as benign lung disease (IPF). The high accuracy and sensitivity are consistent with the effective diagnosis of lung neuroendocrine neoplasia. Gene level measurements were also effective in differentiating between metastatic and localized disease as well as between progressive and stable BPC disease. Overall, the multigene signature test meets minimum biometric performance criteria in terms of sensitivity and specificity [47]. As importantly, the test detects neuroendocrine-derived genes expressed by lung neuroendocrine neoplasia. Any lung nodule that has a positive NETest would likely have a neuroendocrine phenotype and should be considered as a BPC or SCLC. Therefore, the NETest could provide adjunctive information to facilitate histological interpretation. We envisage that the NETest could be used either as a screening tool for lung nodules to help direct follow-up (e.g., subsequent imaging, surgery, or genetic studies of neuroendocrine vs. nonneuroendocrine neoplasia) and for disease monitoring, once the diagnosis has been confirmed. Decreases in scores after surgery could confirm complete tumor removal and, thereafter, be used to monitor residual disease, and, conversely, increasing scores would identify re- currence prior to identification on imaging [17]. Changes in the score could also be used to provide adjunctive information in evaluating responses to therapy, e.g., SSA, as has been reported in gastroenteropancreatic NET [18, 19].

To conclude, this study validates the NETest as a biomarker for BPC. It also provides information that supports the clinical utility of the NETest in the management of lung carcinoids. The data suggest that the NETest may be useful in the differential diagnosis of different lung neoplasias, especially once further algorithmic refinements are further investigated.

\section{Statement of Ethics}

The study was approved by the local ethics committees.

\section{Disclosure Statement}

There are no conflicts of interest that could be perceived as prejudicing the impartiality of the research reported.

\section{Funding Sources}

This work was supported by Yale University, School of Medicine, and the Medical University of Silesia. The Wren Laboratories provided sample measurement at no cost.

\section{References}

1 Gustafsson BI, Kidd M, Chan A, Malfertheiner MV, Modlin IM. Bronchopulmonary neuroendocrine tumors. Cancer. 2008 Jul; 113(1):5-21.

2 Caplin ME, Baudin E, Ferolla P, Filosso P, Garcia-Yuste M, Lim E, et al; ENETS consensus conference participants. Pulmonary neuroendocrine (carcinoid) tumors: European Neuroendocrine Tumor Society expert consensus and recommendations for best practice for typical and atypical pulmonary carcinoids. Ann Oncol. 2015 Aug;26(8):1604-20.

3 Filosso PL, Guerrera F, Thomas P, Brunelli A, Lim E, Garcia-Yuste M, et al.; European Society of Thoracic Surgeons Neuroendocrine Tumors of the Lung Working Group. Management of bronchial carcinoids: international practice survey among the European Society of Thoracic Surgeons. Future Oncol. 2016 Sep;12(17):1985-99.
4 Oberg K, Krenning E, Sundin A, Bodei L, Kidd M, Tesselaar M, et al. A Delphic consensus assessment: imaging and biomarkers in gastroenteropancreatic neuroendocrine tumor disease management. Endocr Connect. 2016 Sep;5(5):174-87.

5 Oberg K, Couvelard A, Delle Fave G, Gross D, Grossman A, Jensen RT, et al; Antibes Consensus Conference participants. ENETS Consensus Guidelines for Standard of Care in Neuroendocrine Tumours: biochemical markers. Neuroendocrinology. 2017;105(3): 201-11.

6 Oberg K, Modlin I, DeHerder W, Pavel M, Klimstra D, Frilling A, et al. Biomarkers for Neuroendocrine Tumor Disease: A Delphic Consensus Assessment of Multianalytes, Genomics, Circulating Cells and Monoanalytes. Lancet Oncol. 2015;16:e435046.

7 Rindi G, Klersy C, Inzani F, Fellegara G, Ampollini L, Ardizzoni A, et al. Grading the neuroendocrine tumors of the lung: an evidencebased proposal. Endocr Relat Cancer. 2014; 21(1):1-16.
8 Tang LH, Gonen M, Hedvat C, Modlin IM, Klimstra DS. Objective quantification of the Ki67 proliferative index in neuroendocrine tumors of the gastroenteropancreatic system: a comparison of digital image analysis with manual methods. Am J Surg Pathol. 2012 Dec; 36(12):1761-70.

9 Pelosi G, Bianchi F, Dama E, Simbolo M, Mafficini A, Sonzogni A, et al. Most high-grade neuroendocrine tumours of the lung are likely to secondarily develop from pre-existing carcinoids: innovative findings skipping the current pathogenesis paradigm. Virchows Arch. 2018 Apr;472(4):567-77.

10 Kidd M, Drozdov I, Modlin I. Blood and tissue neuroendocrine tumor gene cluster analysis correlate, define hallmarks and predict disease status. Endocr Relat Cancer. 2015 Aug;22(4):561-75. 
11 Walenkamp A, Crespo G, Fierro Maya F, Fossmark R, Igaz P, Rinke A, et al. Hallmarks of gastrointestinal neuroendocrine tumours: implications for treatment. Endocr Relat Cancer. 2014;21(6):R445-60.

12 Wang E, Zaman N, McGee S, Milanese JS, Masoudi-Nejad A, O'Connor-McCourt M. Predictive genomics: A cancer hallmark network framework for predicting tumor clinical phenotypes using genome sequencing data. Semin Cancer Biol. 2015 Feb;30:4-12.

13 Oxnard GR, Paweletz CP, Kuang Y, Mach SL, O'Connell A, Messineo MM, et al. Noninvasive detection of response and resistance in EGFR-mutant lung cancer using quantitative next-generation genotyping of cell-free plasma DNA. Clin Cancer Res. 2014 Mar 15; 20(6):1698-705.

14 Fernandez-Cuesta L, Peifer M, Lu X, Sun R, Ozretic L, Seidel D, et al. Frequent mutations in chromatin-remodelling genes in pulmonary carcinoids. Nat Commun. 2014 Mar;5: 3518.

15 Toffalorio F, Belloni E, Barberis M, Bucci G, Tizzoni L, Pruneri G, et al. Gene expression profiling reveals GC and CEACAM1 as new tools in the diagnosis of lung carcinoids. $\mathrm{Br} \mathrm{J}$ Cancer. 2014 Mar;110(5):1244-9.

16 Kidd M, Modlin IM, Drozdov I, Aslanian H, Bodei L, Matar S, et al. A liquid biopsy for bronchopulmonary/lung carcinoid diagnosis. Oncotarget. 2017 Dec;9(6):7182-96.

17 Filosso PL, Kidd M, Roffinella M, Lewczuk A, Chung KM, Kolasinska-Cwikla A, et al. The utility of blood neuroendocrine gene transcript measurement in the diagnosis of bronchopulmonary neuroendocrine tumours and as a tool to evaluate surgical resection and disease progression. Eur J Cardiothorac Surg. 2018 Mar;53(3):631-9.

18 Pavel M, Jann H, Prasad V, Drozdov I, Modlin IM, Kidd M. NET Blood Transcript Analysis Defines the Crossing of the Clinical Rubicon: When Stable Disease Becomes Progressive. Neuroendocrinology. 2017;104(2): $170-82$.

19 Ćwikła JB, Bodei L, Kolasinska-Ćwikła A, Sankowski A, Modlin IM, Kidd M. Circulating transcript analysis (NETest) in GEPNETs treated with somatostatin analogs defines therapy. J Clin Endocrinol Metab. 2015 Nov;100(11):E1437-45.

20 Modlin IM, Drozdov I, Kidd M. The identification of gut neuroendocrine tumor disease by multiple synchronous transcript analysis in blood. PLoS One. 2013 May;8(5):e63364.

21 Modlin IM, Aslanian H, Bodei L, Drozdov I, Kidd M. A PCR blood test outperforms chromogranin A in carcinoid detection and is unaffected by proton pump inhibitors. Endocr Connect. 2014 Dec;3(4):215-23.

22 Zweig MH, Campbell G. Receiver-operating characteristic (ROC) plots: a fundamental evaluation tool in clinical medicine. Clin Chem. 1993 Apr;39(4):561-77.
23 Hanley JA, McNeil BJ. A method of comparing the areas under receiver operating characteristic curves derived from the same cases. Radiology. 1983 Sep;148(3):839-43.

24 Modlin IM, Frilling A, Salem RR, Alaimo D, Drymousis P, Wasan HS, et al. Blood measurement of neuroendocrine gene transcripts defines the effectiveness of operative resection and ablation strategies. Surgery. 2016 Jan;159(1):336-47.

25 Swarts DR, van Suylen RJ, den Bakker MA, van Oosterhout MF, Thunnissen FB, Volante $\mathrm{M}$, et al. Interobserver variability for the WHO classification of pulmonary carcinoids. Am J Surg Pathol. 2014 Oct;38(10):1429-36.

26 Rossi G, Bertero L, Marchiò C, Papotti M. Molecular alterations of neuroendocrine tumours of the lung. Histopathology. 2018 Jan; 72(1):142-52.

27 Liu E, Paulson S, Gulati A, Freudman J, Grosh W, Kafer S, et al. Assessment of NETest Clinical Utility in a U.S. Registry-Based Study. Oncologist. 2018; theoncologist.2017-0623.

28 Alvarez MA, Subramaniam PS, Tang LH, Grunn A, Aburi M, Rieckhof G, et al. A precision oncology approach to the pharmacological targeting of mechanistic dependencies in neuroendocrine tumors.Nat Genet. 2018 Jul; 50(7):979-89.

29 Cui T, Hurtig M, Elgue G, Li SC, Veronesi G, Essaghir A, et al. Paraneoplastic antigen Ma2 autoantibodies as specific blood biomarkers for detection of early recurrence of small intestine neuroendocrine tumors. PLoS One. 2010 Dec;5(12):e16010.

30 Muscarella LA, D'Alessandro V, la Torre A, Copetti M, De Cata A, Parrella P, et al. Gene expression of somatostatin receptor subtypes SSTR2a, SSTR3 and SSTR5 in peripheral blood of neuroendocrine lung cancer affected patients. Cell Oncol (Dordr). 2011 Oct;34(5): $435-41$.

31 Righi L, Volante M, Tavaglione V, Billè A, Daniele L, Angusti T, et al. Somatostatin receptor tissue distribution in lung neuroendocrine tumours: a clinicopathologic and immunohistochemical study of 218 'clinically aggressive’ cases. Ann Oncol. 2010 Mar;21(3): 548-55.

32 Bodei L, Kidd M, Modlin IM, Prasad V, Severi $\mathrm{S}$, Ambrosini V, et al. Gene transcript analysis blood values correlate with ${ }^{68} \mathrm{Ga}$ DOTA-somatostatin analog (SSA) PET/CT imaging in neuroendocrine tumors and can define disease status. Eur J Nucl Med Mol Imaging. 2015 Aug;42(9):1341-52.

33 Lim SB, Tan SJ, Lim WT, Lim CT. A merged lung cancer transcriptome dataset for clinical predictive modeling. Sci Data. 2018 Aug;5: 180136.

34 Tang H, Wang S, Xiao G, Schiller J, Papadimitrakopoulou V, Minna J, et al. Comprehensive evaluation of published gene expression prognostic signatures for biomarker-based lung cancer clinical studies. Ann Oncol. 2017 Apr;28(4):733-40.
35 Chen F, Zhang Y, Gibbons DL, Deneen B, Kwiatkowski DJ, Ittmann M, et al. Pan-Cancer Molecular Classes Transcending Tumor Lineage across 32 Cancer Types, Multiple Data Platforms, and over 10,000 Cases. Clin Cancer Res. 2018 May;24(9):2182-93.

36 Travis WD, Brambilla E, Nicholson AG, Yatabe Y, Austin JH, Beasley MB, et al.; WHO Panel. The 2015 World Health Organization Classification of Lung Tumors: Impact of Genetic, Clinical and Radiologic Advances Since the 2004 Classification. J Thorac Oncol. 2015 Sep;10(9):1243-60.

37 Kosari F, Ida CM, Aubry MC, Yang L, Kovtun IV, Klein JL, et al. ASCL1 and RET expression defines a clinically relevant subgroup of lung adenocarcinoma characterized by neuroendocrine differentiation. Oncogene. $2014 \mathrm{Jul}$; 33(29):3776-83.

38 Cadioli A, Rossi G, Costantini M, Cavazza A, Migaldi M, Colby TV. Lung cancer histologic and immunohistochemical heterogeneity in the era of molecular therapies: analysis of 172 consecutive surgically resected, entirely sampled pulmonary carcinomas. Am J Surg Pathol. 2014 Apr;38(4):502-9.

39 Kadota K, Nitadori J, Rekhtman N, Jones DR, Adusumilli PS, Travis WD. Reevaluation and reclassification of resected lung carcinomas originally diagnosed as squamous cell carcinoma using immunohistochemical analysis. Am J Surg Pathol. 2015 Sep;39(9):1170-80.

40 Feng J, Sheng H, Zhu C, Qian X, Wan D, Su $\mathrm{D}$, et al. Correlation of neuroendocrine features with prognosis of non-small cell lung cancer. Oncotarget. 2016 Nov;7(44):7172736.

41 Trisolini R, Valentini I, Tinelli C, Ferrari M, Guiducci GM, Parri SN, et al. DIPNECH: Association between Histopathology and Clinical Presentation. Hai. 2016 Apr;194(2):243-7.

42 Kidd M, Modlin IM, Mane SM, Camp RL, Eick GN, Latich I, et al. Utility of molecular genetic signatures in the delineation of gastric neoplasia. Cancer. 2006 Apr;106(7):1480-8.

43 Shi J, Li F, Luo M, Wei J, Liu X. Distinct Roles of Wnt/beta-Catenin Signaling in the Pathogenesis of Chronic Obstructive Pulmonary Disease and Idiopathic Pulmonary Fibrosis. Mediators Inflamm. 2017;2017:3520581.

$44 \mathrm{Gu}$ BH, Madison MC, Corry D, Kheradmand F. Matrix remodeling in chronic lung diseases. Matrix Biol. 2018 Nov;73(17):52-63.

$45 \mathrm{He}$ P, Gu X, Wu Q, Lin Y, Gu Y, He J. Pulmonary carcinoid tumorlet without underlying lung disease: analysis of its relationship to fibrosis. J Thorac Dis. 2012 Dec;4(6):655-8.

46 Tamagno G, Goglia U, Villa G, Murialdo G. Lung fibrosis in carcinoid syndrome. Intern Med. 2007;46(7):425-6.

47 Palmer C, Duan X, Hawley S, Scholler N, Thorpe JD, Sahota RA, et al. Systematic evaluation of candidate blood markers for detecting ovarian cancer. PLoS One. 2008 Jul; 3(7):e2633. 